\title{
Automated Quantum Chemistry Based Calculation of Optical Rotation for Large Flexible Molecules
}

\author{
Fabian Bohle ${ }^{\dagger}$, Jakob Seibert ${ }^{\dagger}$ and Stefan Grimme*†
}

September 23, 2021

\section{SUPPORTING INFORMATION}

\section{Contents}

$\begin{array}{lll}1 & \text { Final number of conformers and computation time } & \text { S2 }\end{array}$

2 Comparison and validation of theoretical methods for OR computation S3

3 Computed $[\alpha]_{D}$ for the NROR28 test set for three independent crenso runs S4

4 Solvent information for the NROR28 test set $\quad$ S5

5 Flexibility measure of investigated compounds $\quad$ S6

\begin{tabular}{lll}
\hline 6 & Technical settings applied in the crenso calculations & S7
\end{tabular}

\begin{tabular}{lll}
\hline 7 & Qualitative description of terms and abbreviations & S8
\end{tabular}

8 Short and qualitative methods description $\quad$ S9

\begin{tabular}{llr}
\hline & Statistical means & S10
\end{tabular}

\begin{tabular}{lr}
\hline 10 Geometries & S10
\end{tabular}

\begin{tabular}{lr}
\hline 11 References & $\mathrm{S} 10$
\end{tabular}

${ }^{*}$ Mulliken Center for Theoretical Chemistry, Rheinische Friedrich-Wilhelms-Universität Bonn, Beringstr. 4, 53115, Bonn, Germany

${ }^{\dagger}$ Corresponding author: grimme@thch.uni-bonn.de (phone: +49-228/732544) 


\section{Final number of conformers and computation time}

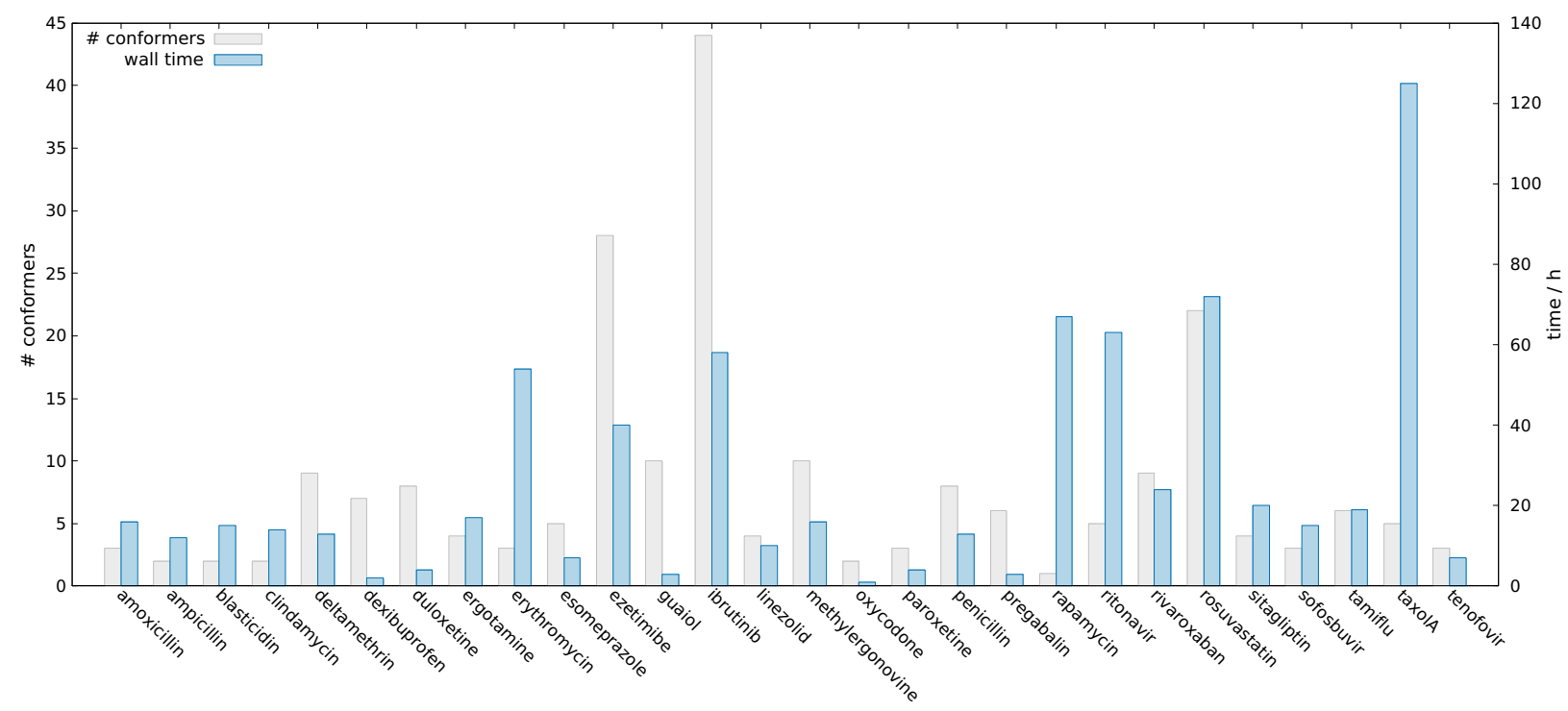

Figure S1: Y-axis1 shows the averaged number of conformers in final ensemble from three independent crenso runs (grey bars) and y-axis2 shows the averaged wall time in hours (blue bars) (Intel ${ }^{\circledR}$ Xeon ${ }^{\circledR}$ CPU E5-2660 v4 @ 2.00GHz at 28 cores) of the NROR28 test set. 


\section{Comparison and validation of theoretical methods for OR computation}

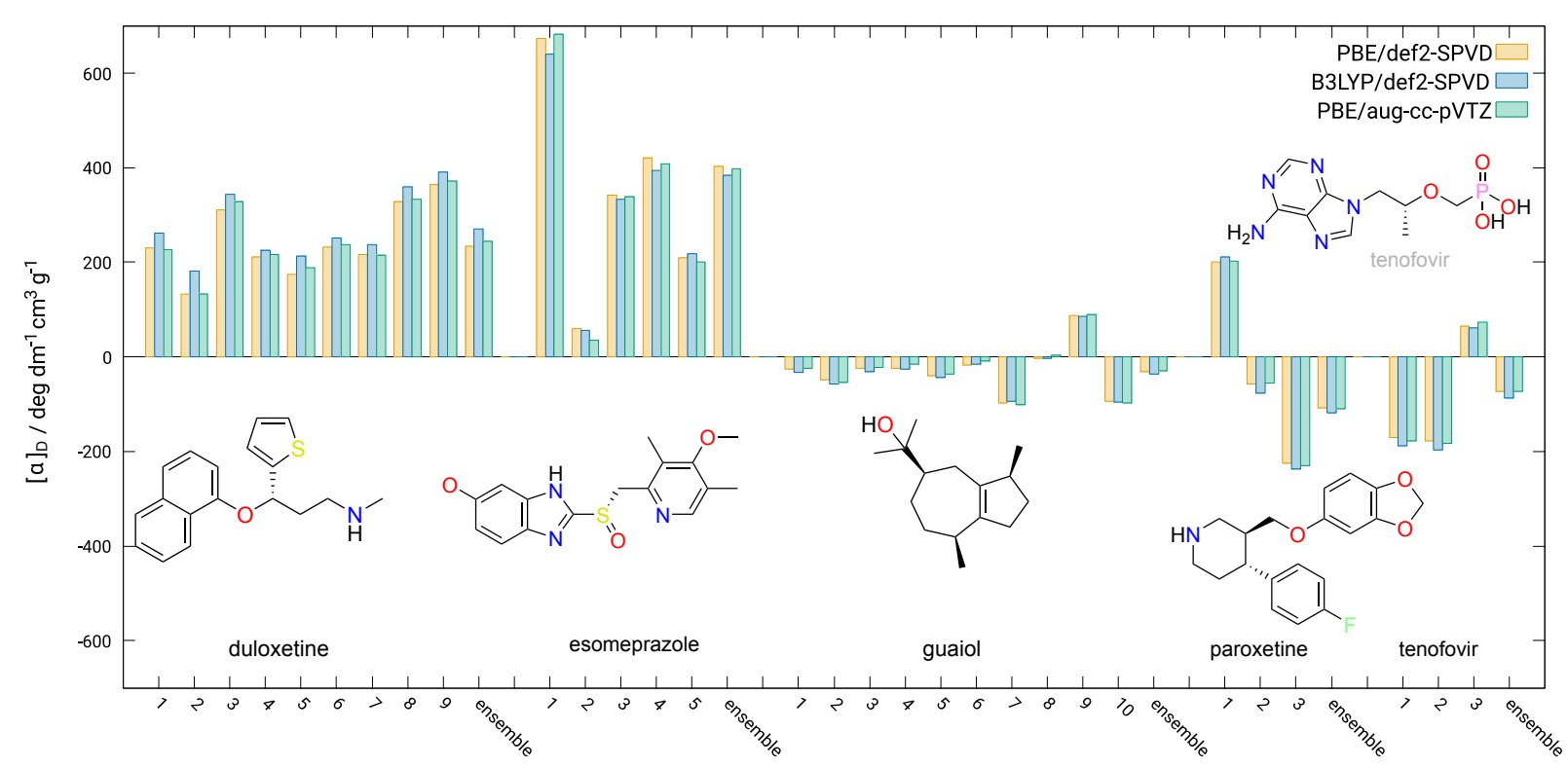

Figure S2: Specific rotation values $\left([\alpha]_{D}\right)$ computed by PBE/def2-SVPD[COSMO] using $\mathrm{r}^{2} \mathrm{SCAN} /$ def2-SVPD[COSMO] orbitals (yellow), B3LYP/def2-SVPD[COSMO] (blue) and PBE/aug-cc-pVTZ[COSMO] using r ${ }^{2} \mathrm{SCAN} /$ def2-SVPD[COSMO] orbitals (green) for a NROR28 subset of five molecules. Solvents are applied as stated in SI-Table1. Numbers refer to individual conformers and the Boltzmann-weighted value is denoted with "ensemble". For all three method combinations within the respective compound the same Boltzmann factors are applied. The B3LYP calculations were performed in the length representation, due to missing implementation of the velocity formalism for hybrid density functionals in TURBOMOLE. 


\section{Computed $[\alpha]_{D}$ for the NROR28 test set for three independent crenso runs}

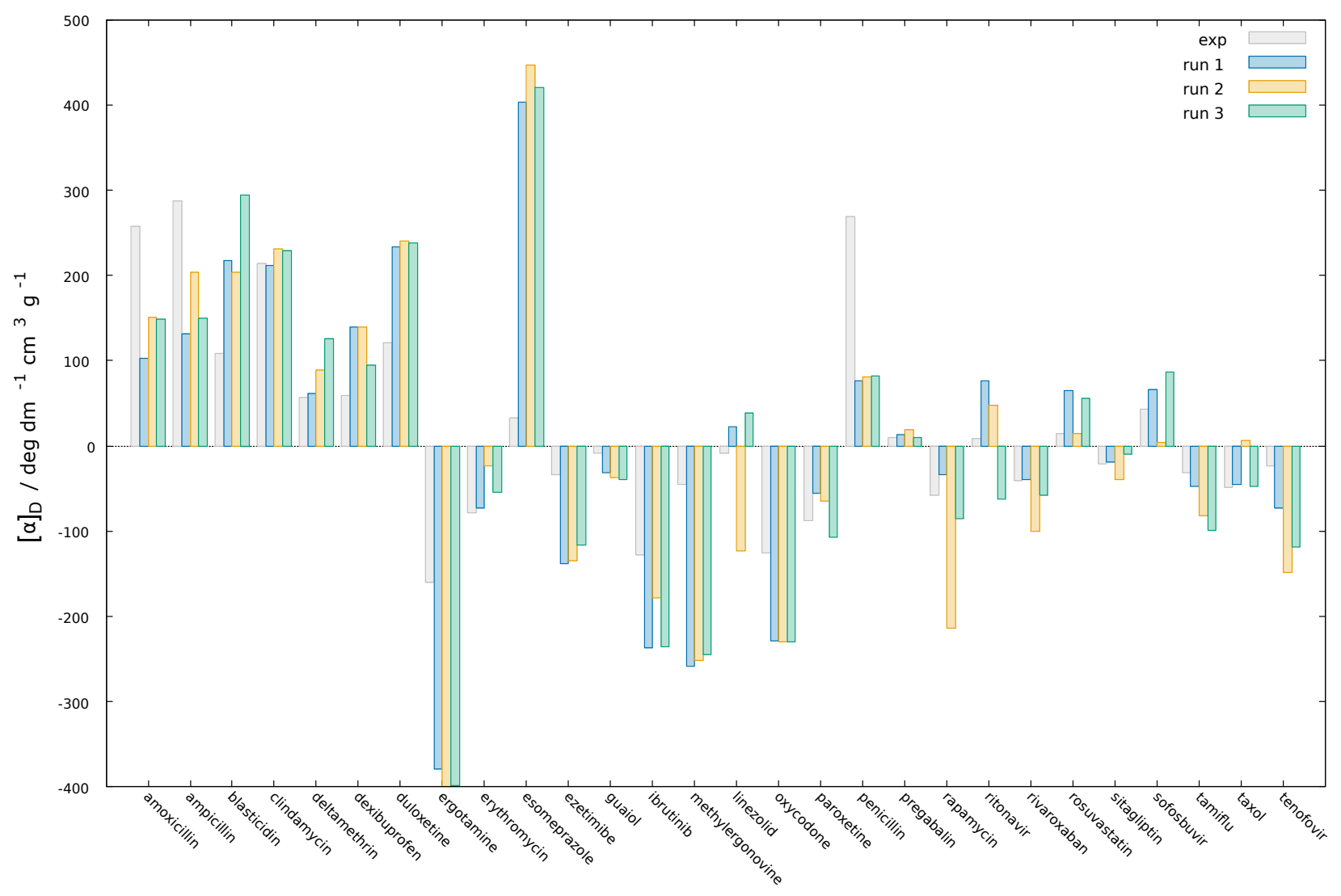

Figure S3: Specific rotation values $\left([\alpha]_{D}\right)$ gathered from experiment $[16$ (grey) and computed from three independent crenso runs (blue, yellow, green) of the NROR28 test set. 


\section{Solvent information for the NROR28 test set}

Table S1: Solvents used in experimental OR measurements and in the calculations. The total charge of the compounds is provided as well.

\begin{tabular}{|c|c|c|c|}
\hline Molecule & solvent, experiment & solvent, theory & total charge \\
\hline amoxicillin & $\mathrm{n} / \mathrm{a}$ & $\mathrm{H}_{2} \mathrm{O}$ & 0 (zwitterionic) \\
\hline ampicillin & $\mathrm{H}_{2} \mathrm{O}$ & $\mathrm{H}_{2} \mathrm{O}$ & 0 (zwitterionic) \\
\hline blasticidin $\mathrm{S}$ & $\mathrm{H}_{2} \mathrm{O}$ & $\mathrm{H}_{2} \mathrm{O}$ & 0 (zwitterionic) \\
\hline clindamycin & $\mathrm{CHCl}_{3}$ & $\mathrm{CHCl}_{3}$ & 0 \\
\hline deltamethrin & Toluene & Toluene & 0 \\
\hline dexibuprofen & $\mathrm{EtOH}$ & $\mathrm{MeOH}$ & 0 \\
\hline duloxetine & $\mathrm{MeOH}$ & $\mathrm{MeOH}$ & 0 \\
\hline ergotamine & $\mathrm{CHCl}_{3}$ & $\mathrm{CHCl}_{3}$ & 0 \\
\hline erythromycin & $\mathrm{EtOH}$ & $\mathrm{MeOH}$ & 0 \\
\hline esomeprazole & $\mathrm{H}_{2} \mathrm{O}$ & $\mathrm{H}_{2} \mathrm{O}$ & -1 \\
\hline ezetimibe & $\mathrm{MeOH}$ & $\mathrm{MeOH}$ & 0 \\
\hline guaiol & $\mathrm{n} / \mathrm{a}$ & $\mathrm{H}_{2} \mathrm{O}$ & 0 \\
\hline ibrutinib & $\mathrm{CHCl}_{3}$ & $\mathrm{CHCl}_{3}$ & 0 \\
\hline linezolid & $\mathrm{CHCl}_{3}$ & $\mathrm{CHCl}_{3}$ & 0 \\
\hline methylergonovine & $\mathrm{n} / \mathrm{a}$ & $\mathrm{H}_{2} \mathrm{O}$ & 0 \\
\hline oxycodone & $\mathrm{H}_{2} \mathrm{O}$ & $\mathrm{H}_{2} \mathrm{O}$ & 0 \\
\hline paroxetine & $\mathrm{EtOH}$ & $\mathrm{MeOH}$ & 0 \\
\hline penicillin & $\mathrm{MeOH}$ & $\mathrm{MeOH}$ & -1 \\
\hline pregabalin & $\mathrm{H}_{2} \mathrm{O}$ & $\mathrm{H}_{2} \mathrm{O}$ & 0 (zwitterionic) \\
\hline rapamycin & $\mathrm{MeOH}$ & $\mathrm{MeOH}$ & 0 \\
\hline ritonavir & $\mathrm{MeOH}$ & $\mathrm{MeOH}$ & 0 \\
\hline rivaroxaban & DMSO & DMSO & 0 \\
\hline rosuvastatin & $\mathrm{MeOH} / \mathrm{H}_{2} \mathrm{O}$ & $\mathrm{MeOH}$ & 0 \\
\hline sitagliptin & $\mathrm{H}_{2} \mathrm{O}$ & $\mathrm{H}_{2} \mathrm{O}$ & +1 \\
\hline sofosbuvir & $\mathrm{EtOH}$ & $\mathrm{MeOH}$ & 0 \\
\hline tamiflu & $\mathrm{H}_{2} \mathrm{O}$ & $\mathrm{H}_{2} \mathrm{O}$ & 0 \\
\hline taxol A & $\mathrm{MeOH}$ & $\mathrm{MeOH}$ & 0 \\
\hline tenofovir & $\mathrm{HCl}(\mathrm{aq})$ & $\mathrm{H}_{2} \mathrm{O}$ & +1 \\
\hline
\end{tabular}




\section{$5 \quad$ Flexibility measure of investigated compounds}

Table S2: The flexibility measure 7$]$ has been extracted from a final crest run in the crenso script. Rigid molecules exhibit flexibility measures close to zero and highly flexible linear alkanes have values of about 0.9. Flexible drug-like molecules show as a rule of thumb measures between 0.3 and 0.5. Larger values for the standard deviation of the ensemble $\mathrm{G}_{m R R H O}$ value indicate a higher structural diversity in the ensemble. The $\mathrm{SD}\left(\mathrm{G}_{m R R H O}\right)$ values have been extracted from the pre-screening filtering part one in censo.

\begin{tabular}{lrrr}
\hline Molecule & no. atoms & Flexibility measure & $\mathbf{S D}\left(\mathbf{G}_{m R R H O}\right)$ \\
\hline amoxicillin & 44 & 0.28 & 0.05 \\
ampicillin & 43 & 0.28 & 0.21 \\
blasticidin S & 56 & 0.25 & 0.42 \\
clindamycin & 60 & 0.35 & 0.20 \\
deltamethrin & 47 & 0.32 & 0.53 \\
dexibuprofen & 33 & 0.34 & 0.18 \\
duloxetine & 40 & 0.41 & 0.29 \\
ergotamine & 78 & 0.23 & 0.47 \\
erythromycin & 118 & 0.30 & 0.51 \\
esomeprazole & 43 & 0.37 & 0.36 \\
ezetimibe & 51 & 0.30 & 0.41 \\
guaiol & 42 & 0.23 & 0.35 \\
ibrutinib & 57 & 0.21 & 0.47 \\
linezolid & 44 & 0.36 & 0.32 \\
methylergonovine & 50 & 0.29 & 0.68 \\
oxycodone & 44 & 0.16 & 0.02 \\
paroxetine & 44 & 0.33 & 0.29 \\
penicillin & 41 & 0.31 & 0.22 \\
pregabalin & 28 & 0.58 & 0.29 \\
rapamycin & 144 & 0.27 & 2.61 \\
ritonavir & 98 & 0.37 & 0.94 \\
rivaroxaban & 47 & 0.30 & 0.63 \\
rosuvastatin & 61 & 0.35 & 0.48 \\
sitagliptin & 43 & 0.33 & 0.65 \\
sofosbuvir & 65 & 0.37 & 0.35 \\
tamiflu & 50 & 0.48 & 0.48 \\
taxol A & 0.27 & 0.66 \\
tenofovir & 0.46 & 0.32 \\
\hline & & &
\end{tabular}




\section{Technical settings applied in the crenso calculations}

Table S3: Technical settings as applied in the crenso calculation using (crenso -or_fine solvent -l3)

\begin{tabular}{|c|c|}
\hline & CENSO general information: \\
\hline CENSO version & 1.0 .8 \\
\hline Temperature & $298.15 \mathrm{~K}$ \\
\hline \multirow[t]{2}{*}{ Solvent } & corresponding to test set system \\
\hline & PART0 - cheap pre-screening \\
\hline Energy & b97-d/def2-SV(P) + GCP \\
\hline Energy_settings & scfconv 5 , grid 1 using D3(0) instead of D3(BJ) \\
\hline G_mRRHO & not included \\
\hline G_Solv & alpb[gfn2] \\
\hline Geometry & GFNn-xTB (input geometry) \\
\hline Threshold & $5.0 \mathrm{kcal} / \mathrm{mol}$ \\
\hline \multirow[t]{2}{*}{ main QM code } & TM \\
\hline & PART1 - pre-screening \\
\hline Energy & r2scan-3c \\
\hline Energy_settings & grid m4 scfconv 6 \\
\hline G_mRRHO & GFN2[alpb]-bhess SPH \\
\hline G_solv & COSMO-RS-normal $[$ r2scan-3c] \\
\hline Geometry & GFNn-xTB (input geometry) \\
\hline Threshold & $3.0 \mathrm{kcal} / \mathrm{mol}$ \\
\hline \multirow[t]{2}{*}{ main QM code } & TM \\
\hline & PART2 - optimization \\
\hline Energy & r2scan-3c \\
\hline Energy_settings & grid $\mathrm{m} 4$ scfconv 6 \\
\hline G_mRRHO & GFN2[alpb]-bhess SPH \\
\hline G_solv & COSMO-RS-normal[r2scan-3c] \\
\hline Geometry & r2scan-3c[DCOSMORS] @optlevel: lax using grid m3 scfconv 6 \\
\hline \multirow{2}{*}{$\begin{array}{l}\text { Threshold } \\
\text { main QM code }\end{array}$} & Opt_limit: $2.5 \mathrm{kcal} / \mathrm{mol}$, Boltzmann sum threshold: $99.0 \%$ \\
\hline & $\frac{\text { TM }}{\text { PART5 - OR mode }}$ \\
\hline Energy & using Energy from part2 - optimization \\
\hline Energy_settings & see part2 - optimization \\
\hline G_mRRHO & using G_mRRHO from part2 - optimization \\
\hline G_solv & using G_solv from part2 - optimization \\
\hline Geometry & r2scan-3c[DCOSMORS] @optlevel: lax \\
\hline Threshold & Boltzmann sum threshold: $99.0 \%$ \\
\hline main QM code & TM \\
\hline Optical rotation & $\begin{array}{c}\left.\text { pbe/def2-SVPD }[\mathrm{COSMO}]_{-}[\mathrm{SCF}=\mathrm{r} 2 \mathrm{scan} / \text { def2-SVPD[COSMO }]\right] \\
\text { using grid } 2 \text { scfconv } 6\end{array}$ \\
\hline
\end{tabular}


Free energies for each conformer are calculated from (see Ref: [8]:

$$
G=E+G_{m R R H O}+\delta G_{\text {solv }}^{T}
$$

Where $\mathrm{E}$ is the electronic energy, $G_{m R R H O}$ represents the thermostatistical contribution to free energy, including the zero point vibrational energy, calculated in the modified rigid rotor harmonic oscillator framework 9,10$]$ and $\delta G_{\text {solv }}^{T}$ is the solvation free energy for the transfer of a compound in the gas phase into solution under standard conditions.

\section{Qualitative description of terms and abbreviations}

$\mathbf{S E}=$ Structural Ensemble refers to a collection of equilibrium geometries (3D-structures) that describes a collection of individual states representing the macroscopic system in the thermodynamic equilibrium. The term "ensemble" originates from statistical mechanics and usually describes a smaller set of possibilities selected from the set of possible states. As such, the term "structural/conformational ensemble" is derived from the canonical ensemble and describes the collection of conformers representing the molecule under the given conditions.

PES = The Potential Energy Surface describes a quantum mechanical system in the Born-Oppenheimer description with its potential energy as a function of the nuclear coordinates. Minima on the PES are for example conformers or rotamers. Dominantly populated conformers decisively describe the macroscopic system. We use the term ConformerRotamer-Ensemble (CRE) for the thermodynamic collection of low (free) energy conformers and rotamers.

protomer $=$ In chemistry, a so-called protomer is a molecule which displays tautomerism due to position of a proton (see: https://en.wikipedia.org/wiki/Protomer).

SQM = Semi-empirical Quantum Mechanical methods are electronic structure methods that are either based on the Hartree Fock or Density Functional Theory formalism, but introduce approximations and often have empirical parameters fitted to specific properties. The approximations are often the neglect of computational expensive parts, e.g., integral evaluations, which speed up the computation significantly compared to ab initio methods. Some examples of SQM methods are: the Hückel method, MNDO, AM1, PMx (x=6,7), DFTB, xTB and many more. Some information can be found in Ref. [11, 12].

Explicit and implicit solvation = In computational chemistry the interaction of the solute with the solvent can be described by mainly two approaches: the implicit (continuum) solvation or "explicit" sovlation involving individual solvent molecules. Continuum models describe the solvent as a uniform polarizable medium with an associated dielectric constant $\epsilon$. Two examples of implicit models are: the CONductor-like Screening MOdel (COSMO) [13] and the Polarizable Continuum Model (PCM)[14]. The explicit treatment of solvation is more involved as individual solvent molecules are included in the quantum 
mechanical calculation. The explicit treatment increases the cost significantly, but also describes all interactions between solute and solvent correctly, within the applied level of theory. However, the minimum number of solvent molecules required to properly solvate the investigated compound is difficult to estimate. In addition the explicit solvent box/cluster around the solute must be equilibrated to represent a system in thermodynamic equilibrium.

\section{Short and qualitative methods description}

xTB: xTB is an eXtended Tight Binding program package. Within xTB semi-empirical quantum mechanical (SQM) methods like GFN1-xTB and GFN2-xTB are implemented. Their target properties are Geometries, vibrational Frequencies and Non covalent interactions. The implemented methods are consistentlly parameterized for all elements up to Radon $(\mathrm{Z}=86)$. Through the use of a minimal atomic orbital (AO) basis set, as well as an efficient Hamiltonian the GFN $n$-xTB methods are at least two orders of magnitude faster, compared with ab initio methods (e.g., DFT). For very fast calculations or when the investigated system size is very large $(\approx 1000$ atoms) a GFN-force field (GFN-FF) is available in xTB. The combination of systematic availability, computational efficiency and robustness makes these methods ideal for the evaluation of chemical space, where millions of single point and gradient calculations have to be performed. Additional information can be obtained from Ref. [12], the online documentation[15] and the code can be obtained from: [16].

crest: The Conformer Rotamer Ensemble Search Tool (CREST) is a sampling program making use of the xtb code, i.e., energies and gradients are calculated at semi-empirical or force field level of theory. It can be used to search the low energy chemical space, and create/analyse conformer, rotamer ensembles (CRE), in other words: identify a conformer distribution for a target compound. In addition, crest can be used to generate protomer or tautomer ensemebles and to find NMR (nuclear exchange) equivalencies. The efficient chemical space exploration is achieved by extensive meta-dynamics (MTD) sampling and information on the use of crest can be obtained from Ref. 17 19.

censo: The Commandline ENergetic SOrting program (CENSO) is used to refine conformer ensembles at higher (DFT) level. Refinement of conformer ensembles in terms of optimized geometries and, in particular, Gibbs free energies is required because the accuracy of SQM methods is not sufficient when very sensitive properties such as optical rotation are studied. CENSO is designed to process large ensembles of conformers, by applying sophisticated combinations of methods (DFT) in terms of computational cost, accuracy and a predefined reasonable choice of sorting thresholds. CENSO interfaces to fast QM codes (TM, ORCA) and analyses the returned results. Additionally, CENSO can provide DFT optimized conformer ensembles, free energies and Boltzmann factors and can be used to calculate specific rotation and NMR properties. Information on the use of CENSO can be obtained from Ref. 20, 21].

crest_combi: crest_combi is a shell script that uses CREST to search conformers on different potential energy surfaces (PES) and combines the results. In this way, a more diverse 
ensemble of conformers can be obtained, i.e., a larger chemical space is explored, than by a single CREST run.

crenso: crenso is a shell script which simplifies the handling of crest and censo for the calculation of properties like $\log \mathrm{P}, \mathrm{NMR}, \mathrm{OR}$, pKa and can be used by simple unix commands e.g. (crenso -l3-or_fine h2o > crenso.out). Its use is explained in Ref. [21] and can be obtained from: https://github.com/grimme-lab/CRENSO.

\section{Statistical means}

MD:

$$
M D=\frac{\sum_{i}^{N} X_{i}}{N}
$$

SD:

$$
S D=\sqrt{\frac{\sum_{i}^{N}\left(X_{i}-M D\right)^{2}}{N-1}}
$$

MAD:

$$
M D=\frac{\sum_{i}^{N}\left|X_{i}\right|}{N}
$$

$\operatorname{relMD}(\%)$ :

$$
\operatorname{rel} M D=\frac{\sum_{i}^{N} \frac{X_{i}-X_{i, r e f}}{0.5 \cdot\left(X_{i, r e f}+X_{i}\right)}}{N} \cdot 100
$$

$\operatorname{relMAD}(\%)$ :

$$
\operatorname{rel} M A D=\frac{\sum_{i}^{N}\left|\frac{X_{i}-X_{i, r e f}}{0.5 \cdot\left(X_{i, r e f}+X_{i}\right)}\right|}{N} \cdot 100
$$

\section{Geometries}

- Atomic Cartesian coordinates (XYZ format, in $\AA$ ) of all input structures and final structure ensembles are provided as structures.zip.

\section{References}

[1] A. Osol, J. T. Anderson, J. E. Hoover, Remington's pharmaceutical sciences, Mack Publishing Company, 1975. 
[2] Agents Classified by the IARC Monographs, Volumes 1-129 - IARC Monographs on the Identification of Carcinogenic Hazards to Humans, monographs . iarc . who . int/ agents-classified-by-the-iarc/, Accessed: 2020-12-20.

[3] The Merck Index: An Encyclopedia of Chemicals, Drugs, and Biologicals, 15th, (Ed.: M. O'Neil), The Royal Society of Chemistry, New Jersey: Merck, 2013.

[4] e-Pesticide Manual, 15th, (Ed.: C. MacBean), British Crop Protection Council, Alton, UK, 2009.

[5] AKSci.com, www.aksci.com, Accessed: 2020-12-20.

[6] chemietek.com, www.chemietek.com, Accessed: 2020-12-20.

[7] P. Pracht, S. Grimme, Chem. Sci. 2021, 12, 6551-6568.

[8] S. Spicher, S. Grimme, J. Phys. Chem. Lett. 2020, 11, 6606-6611.

[9] S. Grimme, Chem. Eur. J. 2012, 18, 9955-9964.

[10] S. Spicher, S. Grimme, Angew. Chem. Int. Ed. 2020, 59, 15665-15673.

[11] A. S. Christensen, T. Kubař, Q. Cui, M. Elstner, Chem. Rev. 2016, 116, PMID: 27074247, 5301-5337.

[12] C. Bannwarth, E. Caldeweyher, S. Ehlert, A. Hansen, P. Pracht, J. Seibert, S. Spicher, S. Grimme, WIREs Comput. Mol. Sci. 2021, 11, DOI 10.1002/wcms.1493.

[13] A. Klamt, G. Schüürmann, J. Chem. Soc. Perkin Trans. 2 1993, 799-805.

[14] B. Mennucci, WIREs Comput. Mol. Sci. 2012, 2, 386-404.

[15] grimme-lab online documentation, https://xtb-docs.readthedocs.io/en/latest/ contents.html.

[16] Grimme-Lab, Grimme-Lab/xtb: Semiempirical extended tight-binding program package, https://github.com/grimme-lab/xtb.

[17] grimme-lab online documentation, https://xtb-docs.readthedocs.io/en/latest/ crest.htm1.

[18] P. Pracht, F. Bohle, S. Grimme, Phys. Chem. Chem. Phys. 2020, 22, 7169-7192.

[19] S. Grimme, J. Chem. Theory Comput. 2019, 15, 2847-2862.

[20] grimme-lab online documentation, https://xtb-docs.readthedocs.io/en/latest/ CENSO_docs/censo.html.

[21] S. Grimme, F. Bohle, A. Hansen, P. Pracht, S. Spicher, M. Stahn, J. Phys. Chem. A 2021, DOI 10.1021/acs.jpca.1c00971. 